\title{
DEPRESSÃO E QUALIDADE DE VIDA EM ADULTOS COM HIPERTENSÃO*
}

\author{
Maria de Fátima Mantovani ${ }^{1}$, Juliana Perez Arthur ${ }^{2}$, Ângela Taís Mattei ${ }^{3}$, Carina Bortolato-Major ${ }^{4}$, Elis \\ Martins Ulbrich ${ }^{5}$ Luciana Puchalski Kalinke ${ }^{6}$
}

\begin{abstract}
RESUMO: O objetivo deste estudo foi investigar a relação entre a depressão e a qualidade de vida em adultos com hipertensão. Pesquisa quantitativa com delineamento transversal, realizada com 387 adultos com hipertensão, no período de 2014 a 2015, em 18 Unidades Básicas de Saúde da cidade de Curitiba, Paraná. Os instrumentos utilizados para avaliar a qualidade de vida e depressão foram, respectivamente, o Medical Outcomes Study 36Item Short Form Health Survey e o inventário de Beck. Observou-se correlação significativa entre os piores escores de qualidade de vida e a presença de depressão, com diferenças elevadas entre as médias nos domínios aspectos emocionais (Intervalo de Confiança $=95 \%: 26,72 \%-42,08 \%$ ) e saúde mental (Intervalo de Confiança $=$ 95\%: 20,21\% - 28,73\%). Conhecer esta relação pode contribuir para o enfermeiro no planejamento do cuidado à pessoa com hipertensão, na melhora dos sintomas depressivos e consequentemente na qualidade de vida.
\end{abstract}

DESCRITORES: Qualidade de vida; Depressão; Hipertensão; Enfermagem.

\section{DEPRESSION AND QUALITY OF LIFE IN HYPERTENSIVE ADULTS}

\begin{abstract}
The present study aimed to investigate the relationship between depression and quality of life in hypertensive adults Quantitative study with cross-sectional design, with 387 adults with hypertension, conducted in 2014-2015, in 18 Basic Health Units (UBS) in the city of Curitiba, State of Paraná. The tools used to assess quality of life and depression were, respectively, the Medical Outcomes Study 36-Item Short Form Health Survey and Beck Inventory. A significant correlation was found between the worst scores of quality of life and the presence of depression, with great differences between the means in the domains emotional aspects (Confidence Interval = 95\%: 26.72\% - 42.08\%) and mental health (Confidence Interval = 95\%: 20.21\% - 28.73\%). Knowledge of this relationship can help nurses in the planning of care for individuals with hypertension, in the improvement of depressive symptoms and quality of life.
\end{abstract}

DESCRIPTORS: Quality of life; Depression; Hypertension; Nursing.

\section{DEPRESIÓN Y CALIDAD DE VIDA EN ADULTOS CON HIPERTENSIÓN}

RESUMEN: El objetivo del estudio fue investigar la relación entre depresión y calidad de vida en adultos con hipertensión. Investigación cuantitativa, con delineado transversal, realizada con 387 adultos con hipertensión entre 2014 y 2015 , en 18 Unidades Básicas e Salud de Curitiba, Paraná. Los instrumentos utilizados para evaluar la calidad de vida y depresión fueron, respectivamente, el Medical Outcomes Study 36-Item Short Form Health Survey y el inventario de Beck. Se observó significativa correlación entre los peores puntajes de calidad de vida y la presencia de depresión, con diferencias elevadas entre las medias en los dominios aspectos emocionales (Intervalo de Confianza $=95 \%: 26,72 \%$ - 42,08\%) y salud mental (Intervalo de Confianza $=95 \%: 20,21 \%-28,73 \%$ ). Conocer dicha relación puede contribuir con el enfermero en la planificación del cuidado al hipertenso, a mejorar los síntomas depresivos y, consecuentemente, en la calidad de vida.

DESCRIPTORES: Calidad de Vida; Depresión; Hipertensión; Enfermería.

\footnotetext{
*Artigo extraído da tese intitulada: "Fatores preditivos para complicações em pessoas com hipertensão arterial sistêmica e ações para o gerenciamento de cuidados da enfermagem na atenção primária". Universidade Federal do Paraná, 2015.

${ }^{1}$ Enfermeira. Doutora em Enfermagem. Coordenadora do Programa de Pós Graduação em Enfermagem da Universidade Federal do Paraná. Curitiba, PR, Brasil.

${ }^{2}$ Enfermeira. Mestranda em Enfermagem. Universidade Federal do Paraná. Curitiba, PR, Brasil.

${ }^{3}$ Enfermeira. Doutoranda em Enfermagem. Universidade Federal do Paraná. Curitiba, PR, Brasil.

${ }^{4}$ Enfermeira. Doutoranda em Enfermagem. Docente da Universidade Estadual do Norte do Paraná. Bandeirantes, PR, Brasil.

${ }^{5}$ Enfermeira. Doutora em Enfermagem. Universidade Federal do Paraná. Curitiba, PR, Brasil.

${ }^{6}$ Enfermeira. Doutora em Enfermagem. Docente de Enfermagem da Universidade Federal do Paraná. Curitiba, PR, Brasil.
}

Autor Correspondente:

Juliana Perez Arthur

Universidade Federal do Paraná

Av. Prefeito Lothário Meissner, 632 - 80210-170 - Curitiba, PR, Brasil

E-mail: julianaperez.4@gmail.com
Recebido: 07/04/2017

Finalizado: 14/08/2017 


\section{INTRODUÇÃO}

A Hipertensão Arterial (HA) é uma doença agressiva, silenciosa, que não possui cura. Ela acomete $32,5 \%$ dos adultos e mais de $60 \%$ dos idosos no Brasil, e tem sido uma das principais causas de mortes por doenças cardiovasculares ${ }^{(1)}$.

No período entre 2008 e 2012, a HA foi responsável por 479.497 internações de brasileiros com mais de 20 anos, o que demandou um gasto acima de 105 milhões de reais nesse período e ocasionou 6.425 óbitos, sendo aproximadamente $20 \%$ na população adulta ${ }^{(2)}$.

Se não controlada, ela pode provocar complicações como o acidente vascular cerebral, infarto agudo do miocárdio, demência, cegueira, insuficiência renal e cardíaca ${ }^{(3)}$, influenciando negativamente na qualidade de vida (QV) das pessoas acometidas, na produtividade do trabalho e na renda familiar ${ }^{(1)}$.

A QV é considerada uma variável subjetiva, que fornece perspectiva multidimensional para avaliação do impacto de aspectos físicos e emocionais na vida de uma pessoa ${ }^{(4)}$, e sua mensuração fornece parâmetros para o planejamento do cuidado. Segundo a Organização Mundial da Saúde, a QV possui um conceito abrangente que envolve aspectos relacionados à saúde física, estado psicológico, nível de independência, relações sociais, crenças pessoais e meio ambiente ${ }^{(5)}$.

Estudos mostram que, em pessoas com hipertensão, a QV pode ser influenciada por aspectos como a idade, estado civil, escolaridade, situação empregatícia, apoio social, tempo de diagnóstico, número de medicamentos e a presença de comorbidades, como a ansiedade e a depressão ${ }^{(4,6-9)}$.

A depressão destaca-se entre estes aspectos por ser um transtorno mental comum capaz de provocar sofrimento ao paciente e a família. Ela se caracteriza pela presença de humor deprimido, perda de interesse ou prazer, diminuição da energia, sentimentos de culpa ou baixa autoestima, distúrbios do sono ou do apetite e falta de concentração ${ }^{(10)}$.

Para pessoas com hipertensão, o efeito da depressão pode gerar uma cascata de ações e resultados negativos, como maior risco de não adesão ao tratamento, inércia clínica e a adoção de hábitos de vida inadequados como o sedentarismo e o tabagismo ${ }^{(11-12)}$, podendo acarretar no descontrole dos níveis pressóricos ${ }^{(13)}$.

A depressão geralmente associa-se a menores pontuações nas avaliações de QV de pessoas com hipertensão, o que se deve principalmente ao seu efeito negativo sobre o humor e possivelmente na capacidade funcional ${ }^{(4,9)}$.

Desta forma, investigar a relação entre a depressão e a QV em pessoas com hipertensão, objetivo desta pesquisa, pode trazer benefícios consideráveis para a tomada de decisão do enfermeiro e demais profissionais de saúde durante a elaboração de um plano de cuidados, tendo em vista a influência destes fatores no seguimento da terapêutica e no desenvolvimento de complicações.

\section{MÉTODO}

Trata-se de uma pesquisa de abordagem quantitativa com delineamento transversal, realizada no período de maio de 2014 a fevereiro de 2015, em 18 Unidades Básicas de Saúde de um Distrito Sanitário do Município de Curitiba, Paraná.

A amostragem estratificada e sistemática foi de 387 adultos que atenderam aos seguintes critérios de inclusão: ter diagnóstico de HA, idade entre 18 a 60 anos, ser cadastrado e ativo no Sistema de Gestão Clínica de Hipertensão Arterial e Diabetes da Unidade Básica de Saúde de referência, e obter pontuação mínima no Mini Exame do Estado Mental ${ }^{(14)}$.

O Mini Exame do Estado Mental tem sua pontuação variando de acordo com a escolaridade. O mínimo requerido são 13 pontos para analfabetos, 18 para pessoas com baixa e média escolaridade e 26 para aqueles com alta escolaridade. Pessoas que possuem até oito anos incompletos de estudo são consideradas com baixa e média escolaridade e acima disso com alta escolaridade ${ }^{(15)}$. 
Para a coleta de dados, todos os pesquisadores foram treinados para padronização da abordagem. Foi utilizado um questionário semiestruturado com variáveis sociodemográficas, os instrumentos Medical Outcomes Study 36-Item Short Form Health Survey (SF- 36) e o inventário de depressão de Beck.

O SF-36 é um instrumento de avaliação da QV multidimensional, traduzido e adaptado transculturalmente para a língua portuguesa ${ }^{(16)}$. Contém 36 itens englobados em oito domínios: estado geral de saúde, limitação por aspectos físicos, dor, capacidade funcional, aspectos emocionais, vitalidade, aspectos sociais e saúde mental ${ }^{(17)}$. Possui um escore de zero a 100, a pontuação máxima representa um melhor estado de saúde e a mínima um pior estado de saúde.

O inventário de depressão de Beck foi validado para a língua portuguesa e permite analisar a intensidade da depressão. Possui 21 itens que descrevem as manifestações comportamentais cognitivas afetivas e somáticas da depressão ${ }^{(18)}$. A interpretação de seus resultados é realizada em quatro graus de intensidade: sem depressão ou depressão mínima (menos do que 10 pontos); depressão leve a moderada (de dez a 18); depressão moderada a grave (de 19 a 29) e depressão grave (de 30 a 63) ${ }^{(18)}$.

A fim de facilitar a análise dos dados, optou-se por agrupar os graus de intensidade em duas categorias. As pessoas que obtiveram pontuações até nove foram consideradas "sem depressão" e aquelas com pontuação maior ou igual a 10 "com depressão". Os dados foram tabulados em planilhas do Microsoft Excel ${ }^{\otimes}$, analisados por estatística descritiva, e a correlação entre QV e depressão foi realizada com o teste T-Student para amostras independentes, utilizando o software Statistical Package for Social Sciences (SPSS) versão 20.0. Para verificar a normalidade dos dados utilizou-se o teste de Kolmogorov-Smirnov. O nível de significância foi de 5\%.

Esta pesquisa faz parte do projeto temático intitulado "Construção e validação de instrumento para o cuidado da hipertensão arterial sistêmica e as representações de cuidado de adultos hipertensos". Foi aprovado pelo Comitê de Ética em Pesquisa do Setor de Ciências da Saúde da Universidade Federal do Paraná sob parecer 220.068 em 15 de março de 2013.

\section{RESULTADOS}

Das 387 pessoas estudadas, 295 (76,25\%) eram do sexo feminino, 266 (68,8\%) eram casadas ou em união consensual, 135 (34,9\%) possuíam mais de oito anos de estudo e 202 (52,3\%) tinham renda familiar entre um e três salários mínimos (Tabela 1).

Tabela 1 - Características sócio demográficas dos participantes da pesquisa (n=387). Curitiba, PR, Brasil, 2016 (continua)

\begin{tabular}{lc}
\hline VARIÁVEL & n (\%) \\
\hline Sexo & \\
\hline Masculino & $92(23,8)$ \\
\hline Feminino & $295(76,2)$ \\
\hline Estado Conjugal & $52(13,4)$ \\
\hline Solteiro(a) & $266(68,8)$ \\
\hline Casado(a)/união consensual & $34(8,8)$ \\
\hline Divorciado(a)/Separado(a) & $35(9)$ \\
\hline Viúvo(a) & $14(3,6)$ \\
\hline Anos de estudo & $130(33,6)$ \\
\hline Sem estudo & $108(27,9)$ \\
\hline 1 a 4 & $135(34,9)$ \\
\hline 5 a 8 & \\
\hline Acima de 8 &
\end{tabular}




\begin{tabular}{lc}
\hline Renda Familiar (salários mínimos)* $^{*}$ & \\
\hline Não possui renda & $1(0,3)$ \\
\hline$<1$ & $18(4,7)$ \\
\hline 1 a 3 & $202(52,3)$ \\
\hline 4 a 5 & $120(31)$ \\
\hline$>5$ & $45(11,7)$
\end{tabular}

*Salário equivalente a 724,00 reais.

A frequência de participantes com depressão foi de 168 (43,4\%). Em relação aos resultados da QV com o questionário SF-36, os participantes apresentaram as médias mais baixas nos seguintes domínios: dor, vitalidade, estado geral de saúde e aspectos físicos. A melhor pontuação média foi para o domínio aspectos sociais (Tabela 2).

Tabela 2 - Média, mediana e desvio padrão dos valores obtidos para os domínios do SF-36. Curitiba, PR, Brasil, 2016

\begin{tabular}{lccc} 
Domínio do SF-36 & Média & Mediana & Desvio padrão \\
\hline Capacidade Funcional & 73,2 & 80 & 25,3 \\
\hline Aspectos Físicos & 65,8 & 100 & 41,3 \\
\hline Dor & 58,8 & 61 & 28 \\
\hline Estado Geral de Saúde & 64,8 & 72 & 23,2 \\
\hline Vitalidade & 63,8 & 65 & 22,3 \\
\hline Aspectos Sociais & 77,9 & 88 & 25,8 \\
\hline Aspectos Emocionais & 73 & 100 & 40 \\
\hline Saúde Mental & 70,3 & 76 & 23,5
\end{tabular}

Ao comparar os escores dos domínios do SF-36 entre participantes com depressão e sem depressão, verificou-se que as pontuações foram significativamente menores em todos os domínios para pessoas com depressão. As maiores diferenças entre as médias foram encontradas nos aspectos emocionais (IC=95\%: 26,72\% - 42,08\%), saúde mental (IC=95\%: 20,21\% - 28,73\%), aspectos físicos (IC=95\%: 15,85\% - 32,10\%) e vitalidade (IC=95\%: 19,14\% - 27,07\%) (Tabela 3).

Tabela 3 - Comparação entre as médias obtidas nos domínios do SF-36, de acordo com a classificação na Escala de Depressão de Beck. Curitiba, PR, 2016 (continua)

\begin{tabular}{|c|c|c|c|c|}
\hline \multirow[t]{3}{*}{ Domínios SF-36 } & \multicolumn{2}{|c|}{$\begin{array}{c}\text { Classificação na escala de Depressão } \\
\text { de Beck }\end{array}$} & \multirow[t]{3}{*}{$\begin{array}{l}\text { Diferença entre } \\
\text { médias (IC 95\%) }\end{array}$} & \multirow[t]{3}{*}{ p-valor } \\
\hline & Sem depressão $^{a}$ & Depressão $^{b}$ & & \\
\hline & Média (DP) & Média (DP) & & \\
\hline Capacidade funcional & $79,38(23,33)$ & $65,12(25,55)$ & $14,26(9,30$ a 19,23$)$ & $<0,01$ \\
\hline Aspectos físicos & $76,26(36,65)$ & $52,23(43,24)$ & $24,02(15,85$ a 32,10$)$ & $<0,01$ \\
\hline Dor & $65,65(27,44)$ & $49,77(26,31)$ & $15,88(10,44$ a 21,31$)$ & $<0,01$ \\
\hline Estado de saúde geral & $71,29(19,81)$ & $56,40(24,53)$ & $14,89(10,33$ a 19,45$)$ & $<0,01$ \\
\hline Vitalidade & $73,79(17,45)$ & $50,68(21,21)$ & $23,10(19,14$ a 27,07$)$ & $<0,01$ \\
\hline Aspectos sociais & $86,36(20,71)$ & $66,96(27,67)$ & $19,39(14,37$ a 24,42$)$ & $<0,01$ \\
\hline Aspectos emocionais & $87,98(29,48)$ & $53,57(43,47)$ & $34,40(26,72$ a 42,08$)$ & $<0,01$ \\
\hline Saúde mental & $80,88(16,34)$ & $56,40(24,13)$ & $24,72(20,21$ a 28,73$)$ & $<0,01$ \\
\hline
\end{tabular}


estatístico: T-Student amostras independentes.

Fonte: o autor (2016)

\section{DISCUSSÃO}

O presente estudo apresentou três achados principais. O primeiro se refere à porcentagem de adultos com hipertensão que possuíam depressão, o segundo são os domínios de QV mais afetados, e o terceiro a possível relação entre a diminuição da QV e a presença de depressão em pessoas com hipertensão.

Os aspectos sociodemográficos da amostra encontrados nesta pesquisa corroboram com aqueles da literatura nacional quanto às características das pessoas com $\mathrm{HA}^{(1)}$. Quanto à $\mathrm{QV}$, sabe-se que ela está associada ao nível de escolaridade, e, embora esta comparação não tenha sido realizada, ela pode ter influenciado nos escores do SF-36, pois verificou-se que 33,6\% dos participantes possuíam de um a quatro anos de estudo(6).

A variável depressão também pode ser avaliada relacionando-se com os anos de estudo, entretanto, ao verificar esta associação, não há um consenso estabelecido na literatura, pois os dados são heterogêneos e dependem da população investigada ${ }^{(19)}$.

A presença da depressão em pessoas com hipertensão, encontrada nesta pesquisa, também tem sido identificada em estudos internacionais conduzidos a partir de metodologias, populações e amostragens distintas ${ }^{(20-21)}$.

Revisão sistemática com meta-análise de estudos observacionais que utilizou as bases de dados PubMed, Web of Knowledge, China National Knowledge, Internet (CNKI), WanFang, e Weipu cujo objetivo foi verificar a prevalência de depressão em pessoas com hipertensão, identificou 41 estudos com um total de 30.796 pessoas e apontou que a prevalência média de pacientes com hipertensão que possuíam depressão foi de 26,8\% (IC=95\%: 21.7\%-32.3\%). Do total de estudos incluídos na revisão, $80 \%$ era de corte transversal, o mesmo empregado nesta pesquisa ${ }^{(21)}$.

Ao investigar a ansiedade e depressão em 204 pacientes com doenças crônicas, na Grécia, os autores verificaram que $36,3 \%$ da amostra possuía hipertensão e $54 \%$ algum grau de depressão ${ }^{(22)}$. Por outro lado, em 2014, no estado de Minas Gerais, Brasil, foi realizado um estudo transversal com 172 idosos com HA que encontrou sintomas de depressão em $30,2 \%$ da amostra ${ }^{(23)}$.

A média de pessoas com HA e depressão mostrou-se superior àquelas encontradas nos estudos descritos anteriormente. Entretanto, conforme a meta-análise que investigou essa relação, a heterogeneidade da prevalência da depressão em pacientes com hipertensão pode ser atribuída aos diferentes métodos de avaliação ${ }^{(21)}$, tendo em vista que quando os instrumentos são auto declarados pode haver uma superestimação ou subestimação dos resultados.

Os sintomas da depressão podem afetar a $\mathrm{QV}^{(24)}$, a adesão ao tratamento medicamentoso e não medicamentoso de pessoas com hipertensão, além de predispor a hábitos inadequados, devido principalmente as alterações de humor que ela ocasiona ${ }^{(11)}$, podendo predispor ao aparecimento de complicações.

A relação entre a depressão e o controle da hipertensão não foi avaliada nesta pesquisa, porém, acredita-se que pessoas com depressão possuem maiores dificuldades no seguimento da terapêutica, fato demonstrado em um estudo de caso-controle desenvolvido no Paquistão com o objetivo de investigar a associação entre hipertensão não controlada com transtornos depressivos e de ansiedade. Nele, foram incluídos 590 pacientes que se apresentaram em dois ambulatórios com pressão arterial acima de 140/90 mmHg. Os resultados demonstraram que houve associação entre hipertensos não controlados e história de depressão ${ }^{(24)}$.

Em estudo realizado no Rio Grande do Sul, Brasil, com 302 pacientes para verificar a associação entre sintomas de ansiedade ou depressão, HA e seu impacto na QV, utilizando-se os questionários de Beck e o SF-36, demonstrou que a depressão e a ansiedade influenciam negativamente na QV ${ }^{(25)}$. 
$\mathrm{Na}$ presente pesquisa encontraram-se resultados similares ao estudo anterior entre os escores de QV obtidos por pessoas com e sem depressão, em todos os domínios. Mesmo sendo um estudo transversal, que não permite estabelecer uma relação de causa-efeito, é possível inferir que a depressão influenciou negativamente na QV dos pacientes investigados.

As pesquisas que relacionam a QV de hipertensos com depressão são escassas, mas com outras doenças crônicas como o diabetes e a doença renal crônica essa associação pode ser encontrada.

Estudo transversal realizado no norte do Irã com o objetivo de verificar a relação entre a QV e a depressão em pessoas com diabetes tipo II, utilizou como instrumento de avaliação o WHO Quality of Life-BREF (WHOQOL-BREF). Os resultados apontaram que a média da QV dos pacientes com depressão foi significativamente menor em três (saúde física, saúde psicológica e ambiente) dos quatro domínios do instrumento, quando comparada aos individuos que não possuem ${ }^{(26)}$.

Embora o instrumento utilizado para a avaliação da QV no estudo citado anteriormente não seja o SF-36, nota-se que a diferença entre as pontuações das pessoas com e sem depressão para o domínio Saúde Psicológica do WHOQOL-BREF foi significativo $(p<0,001)$, resultado semelhante ao encontrado nesta pesquisa para os domínios Saúde Mental $(p<0,01)$ e Aspectos emocionais $(p<0,01)$.

Corroborando com estes resultados, um estudo de coorte realizado com 198 pacientes com diabetes tipo II, em Timisoara, Romênia, aponta a depressão como fator capaz de influenciar na diminuição da QV de pessoas com diagnóstico de diabetes, sendo essa influência intensificada de acordo com a gravidade do quadro depressivo ${ }^{(27)}$.

Em relação à doença renal crônica, um estudo publicado em 2016, do tipo correlacional, de corte transversal, desenvolvido em uma Unidade de Terapia Renal Substitutiva do interior do Estado de São Paulo, Brasil, associou a ansiedade e a depressão com a diminuição da QV dos pacientes com esta patologia e que faziam hemodiálise ${ }^{(28)}$.

Estudo realizado na Índia com adultos diagnosticados com doença pulmonar obstrutiva crônica grave e muito grave, mostrou que os pacientes com depressão grave obtiveram escore mais baixo de QV em todos os domínios em comparação com aqueles indivíduos com depressão moderada ou leve, além disso, o número de exacerbações e hospitalizações da doença tiveram correlação com a gravidade da depressão e com todos os domínios da QV ${ }^{(29)}$.

Portanto, estudos que relacionam QV e depressão, mesmo em diferentes doenças crônicas não transmissíveis, apresentam resultados similares a estes e apontam para outras repercussões que não foram avaliadas.

A limitação desta pesquisa refere-se ao tipo de estudo com corte transversal, fato que não possibilita estabelecer uma relação de causa e efeito.

\section{CONSIDERAÇÕES FINAIS}

Esta pesquisa sugere que a depressão está significativamente associada a pior QV em pacientes com hipertensão em todos os domínios do SF-36.

Tendo em vista que essa relação pode afetar o seguimento da terapêutica medicamentosa e não medicamentosa, faz-se necessário que o enfermeiro, bem como a equipe multiprofissional, conheça a pessoa com hipertensão e avalie seu estado de saúde para juntos estabelecerem intervenções individualizadas e efetivas, que os auxiliem na melhora dos sintomas depressivos e consequentemente na QV, no entanto, faltam evidências científicas fortes para comprovar essa relação e com isso auxiliar os profissionais na tomada de decisão.

\section{- AGRADECIMENTOS}

Ao Conselho Nacional de Desenvolvimento Científico e Tecnológico pela concessão da bolsa produtividade (2014/2017) à autora principal e pelo financiamento Edital Universal- 2012-2015. 
À Fundação Araucária de Apoio ao Desenvolvimento Científico e Tecnológico do Estado do Paraná pela concessão do financiamento Edital 2014-2016.

\section{- REFERÊNCIAS}

1. Malachias MVB, Souza WKSB, Plavnik FL, Rodrigues CIS, Brandão AA, Neves MFT, et al. $7^{\text {a }}$ Diretriz Brasileira de Hipertensão Arterial. Arq Bras Cardiol. [Internet] 2016;107(3 Suppl 3) [acesso em 15 nov 2016]. Disponível: http:// publicacoes.cardiol.br/2014/diretrizes/2016/05_HIPERTENSAO_ARTERIAL.pdf.

2. Santos SS, de Vasconcelos DFSA. Hospitalizations for essential hypertension urgency in Brazil, 2008-2012. Rev. Ciênc. Méd. Biol. [Internet] 2013;12(n.esp) [acesso em 04 nov 2016]. Disponível: https://portalseer.ufba.br/index. php/cmbio/article/viewFile/9191/6758.

3. World Health Organization (WHO). Global status report on noncommunicable diseases. [Internet] Geneva: World Health Organization; 2014 [acesso em 15 nov 2016]. Disponível: http://apps.who.int/iris/ bitstream/10665/148114/1/9789241564854_eng.pdf?ua=1.

4. Baladón L, Rubio-Valera M, Serrano-Blanco A, Palao DJ, Fernández A. Gender differences in the impact of mental disorders and chronic physical conditions on health-related quality of life among non-demented primary care elderly patients. Qual Life Res. [Internet] 2016;25(6) [acesso em 22 fev 2017]. Disponível: https://dx.doi. org/10.1007/s11136-015-1182-5.

5. World Health Organization (WHO). Promoción de la salud: glosario. [Internet] Genebra: WHO; 1998 [acesso em 20 fev 2017]. Disponível: https://www.msssi.gob.es/profesionales/saludPublica/prevPromocion/docs/glosario. pdf.

6. Zhang Y, Zhou Z, Gao J, Wang D, Zhang Q, Zhiying Z, et al. Health-related quality of life and its influencing factors for patients with hypertension: evidence from the urban and rural areas of Shaanxi Province. China. BMC Health Serv Res. [Internet] 2016;(16) [acesso em 22 fev 2017]. Disponível: https://dx.doi.org/10.1186/s12913-0161536-x.

7. de Carvalho MV, Siqueira LB, Sousa ALL, Jardim PCBV. The influence of hypertension on quality of life. Arq. Bras. Cardiol. [Internet] 2013;100(2) [acesso em 25 mar 2017]. Disponível: http://dx.doi.org/10.5935/abc.20130030.

8. Xu X, Rao Y, Shi Z, Liu L, Chen C, Zhao Y. Hypertension Impact on Health-Related Quality of Life: A CrossSectional Survey among Middle-Aged Adults in Chongqing, China. Int J Hypertens. [Internet] 2016;(7404957) [acesso em 23 mar 2017]. Disponível: http://dx.doi.org/10.1155/2016/7404957.

9. Khalifeh M, Salameh P, Hajje AA, Awada S, Rachidi S, Bawab W. Hypertension in the Lebanese adults: Impact on health related quality of life. J Epidemiol Glob Health. [Internet] 2015;5(4) [acesso em 23 mar 2017]. Disponível: http://dx.doi.org/10.1016/j.jegh.2015.02.003.

10. World Health Organization (WHO). Depression: A Global Crisis. World Federation for Mental Health. [Internet] Geneva: WHO; 2012 [acesso em 25 nov 2016] Disponível: http://www.who.int/mental_health/management/ depression/wfmh_paper_depression_wmhd_2012.pdf?ua=1.

11. Moise N, Davidson KW, Chaplin W, Shea S, Kronish I. Depression and clinical inertia in patients with uncontrolled hypertension. JAMA Intern Med. [Internet] 2014;174(5) [acesso em 23 mar 2017]. Disponível: http:// dx.doi.org/10.1001/jamainternmed.2014.115.

12. Bautista LE, Vera-Cala LM, Colombo C, Smith P. Symptoms of depression and anxiety and adherence to antihypertensive medication. Am J Hypertens. [Internet] 2012;25(4) [acesso em 25 nov 2016]. Disponível: https:// doi.org/10.1038/ajh.2011.256.

13. Rubio-Guerra AF, Rodriguez-Lopez L, Vargas-Ayala G, Huerta-Ramirez S, Serna DC, Lozano-Nuevo JJ. Depression increases the risk for uncontrolled hypertension. Exp Clin Cardiol. [Internet] 2013;18(1) [acesso em 14 mar 2017]. Disponível: https://www.ncbi.nlm.nih.gov/pmc/articles/PMC3716493/.

14. Folstein MF, Folstein SE, McHugh PR. "Mini-mental state". A practical method for grading the cognitive state of patients for the clinician. J Psychiatr Res. [Internet] 1975;12(3) [acesso em 14 nov 2016]. Disponível: http:// dx.doi.org/10.1016/0022-3956(75)90026-6. 
15. Crum RM, Anthony JC, Bassett SS, Folstein MF. Population-Based Norms for the Mini-Mental State Examination by Age and Educational Level. JAMA. [Internet] 1993;269(18) [acesso em 14 nov 2016]. Disponível: http://dx.doi. org/10.1001/jama.1993.03500180078038.

16. Ciconelli RM, Ferraz MB, Santos W, Meinão I, Quaresma MR. Tradução para a língua portuguesa e validação do questionário genérico de avaliação da qualidade de vida SF-36 (Brasil-SF-36). Rev. Bras Reumatol. [Internet] 1999;39(3) [acesso em 14 nov 2016]. Disponível: http://www.ufjf.br/renato_nunes/files/2014/03/ Valida\%C3\%A7\%C3\%A3o-do-Question\%C3\%A1rio-de-qualidade-de-Vida-SF-36.pdf.

17. Ware JE, Sherbourne CD. The MOS 36-item short-form health survey (SF-36). I. Conceptual framework and item selection. Med Care. [Internet] 1992;30(6) [acesso em 14 nov 2016]. Disponível: https://www.ncbi.nlm.nih. gov/pubmed/1593914.

18. Beck AT, Steer RA, Carbin MG. Psychometric properties of the Beck Depression Inventory: Twenty five years of evaluation. Clin Psychol Rev. [Internet] 1988;8(1) [acesso em 14 nov 2016]. Disponível: https://doi. org/10.1016/0272-7358(88)90050-5.

19. Bauldry S. Variation in the Protective Effect of Higher Education against Depression. Society and Mental Health. [Internet] 2015;5(2) [acesso em 06 abr 2017]. Disponível: http://journals.sagepub.com/doi/ full/10.1177/2156869314564399.

20. Kabutoya T, Kario K. Depression in hypertension and blood pressure variability over shorter time periods. Hypertens Res. [Internet] 2015;38(11) [acesso em 06 abr 2017]. Disponível: http://dx.doi.org/10.1038/hr.2015.92.

21. Li Z, Li Y, Chen L, Chen P, Hu Y. Prevalence of Depression in Patients With Hypertension: a Systematic Review and Meta-Analysis. Medicine. [Internet] 2015;94(31) [acesso em 23 mar 2017]. Disponível: http://dx.doi. org/10.1097/MD.0000000000001317.

22. Gerontoukou E-I, Michaelidoy S, Rekleiti M, Saridi M, Souliotis K. Investigation of Anxiety and Depression in Patients with Chronic Diseases. Health Psychol Res. [Internet] 2015;3(2) [acesso em 25 fev 2017]. Disponível: http://dx.doi.org/10.4081/hpr.2015.2123.

23. da Silva PCS, Monteiro LA, Graciano ADS, Terra FS, Veiga EV. Assessment of depression in elderly with systemic hypertension. Rev Rene. [Internet] 2014;15(1) [acesso em 11 mar 2017]. Disponível: http://www.revistarene.ufc.br/ revista/index.php/revista/article/view/1570.

24. Almas A, Patel J, Ghori U, Ali A, Edhi AI, Khan MA. Depression is linked to uncontrolled hypertension: a casecontrol study from Karachi, Pakistan. J Ment Health. [Internet] 2014;23(6) [acesso em 20 fev 2017]. Disponível: http://dx.doi.org/10.3109/09638237.2014.924047.

25. Saboya PM, Zimmermann PR, Bodanese LC. Association between anxiety or depressive symptoms and arterial hypertension and their impact on the quality of life. Int J Psychiatry Med. [Internet] 2010;40(3) [acesso em 25 mar 2017]. Disponível: http://journals.sagepub.com/doi/10.2190/PM.40.3.f.

26. Derakhshanpour F, Vakili MA, Farsinia M, Mirkarimi K. Depression and Quality of Life in Patients With Type 2 Diabetes. Iran Red Crescent Med J. [Internet] 2015;17(5) [acesso em 25 mar 2017]. Disponível: http://dx.doi. org/10.5812/ircmj.17(5)2015.27676.

27. Timar R, Velea I, Timar B, Lungeanu D, Oancea C, Roman D, et al. Factors influencing the quality of life perception in patients with type 2 diabetes mellitus. Patient Prefer Adherence. [Internet] 2016;(10) [acesso em 25 mar 2017]. Disponível: https://doi.org/10.2147/PPA.S124858.

28. Ottaviani AC, Betoni LC, Pavarini SCI, Say KG, Zazzetta MS, Orlandi FS. Association between anxiety and depression and quality of life of chronic renal patients on hemodialysis. Texto Contexto Enferm. [Internet] 2016;25(3) [acesso em 25 mar 2017]. Disponível: http://dx.doi.org/10.1590/0104-07072016000650015.

29. Channareddy LR, Ravula ER, Kumar V. Depression and quality of life in patients with severe chronic obstructive pulmonary disease -A cross sectional study. IAIM. [Internet] 2016;3(4) [acesso em 14 mar 2017]. Disponível http:// iaimjournal.com/wp-content/uploads/2016/04/iaim_2016_0304_11.pdf. 